\title{
Clinical profile, visual outcome and complications after cataract surgery in patients with lens induced glaucoma
}

\author{
Sangeetha Jayakumar' ${ }^{1}$, Kanthamani Krishnappa ${ }^{2, *}$, Darshan $\mathrm{SM}^{3}$, Chirra Reddy Gari Meghana ${ }^{4}$, \\ Borra Harish Laxman ${ }^{5}$ \\ ${ }^{1}$ Assistant Professor, ${ }^{2}$ Professor, ${ }^{3}$ Senior Resident, ${ }^{4,5}$ Junior Resident, Sri Devaraj Urs Medical College, Kolar, Karnataka, India \\ *Corresponding Author: Kanthamani Krishnappa \\ Email: dr.kanthamanik@gmail.com
}

\begin{abstract}
Purpose: To assess the clinical profile of lens-induced glaucoma (LIG), reasons for late presentation, and outcome of medical and surgical management.

Materials and Methods: Retrospective analysis of 48 eyes with LIG over a 5-year period between 2014 and 2018 at a tertiary care centre in rural Southern India. Type of LIG, symptoms, preoperative and postoperative corrected distant visual acuity (CDVA), intraocular pressure (IOP), intraoperative and postoperative complications were assessed.

Results: Forty-eight eyes of 23(47.9\%) males and 25(52.1\%) female aged between 50 to 80 years were included in this study. $32(66.7 \%)$ cases had presented with phacomorphic glaucoma and $13(27.1 \%)$ with Phacolytic glaucoma who underwent medical and surgical treatment of the same. Mean pre-operative and postoperative IOP was $35.60 \pm 5.81 \mathrm{mmHg}$ and $20.417 \pm 2.33$ respectively. Similarly, the mean preoperative and postoperative CDVA was $1.44 \pm 0.76$ and $0.34 \pm 0.20$ respectively which was statistically significant. ( $\mathrm{P}<0.001$ ). Most commonly observed complications were shallow anterior chamber, striae keratopathy and iritis that resolved with prompt postoperative treatment.

Conclusion: LIG is an important vision-threatening disease occurring in the elderly due delayed treatment of mature cataract. This study shows that it is important to conduct periodic screening of the elderly to identify cataracts and manage it accordingly to prevent irreversible blindness.
\end{abstract}

Keywords: Intraocular pressure, Glaucoma, Phacomorphic, Phacolytic.

\section{Introduction}

Cataract is the most important cause of treatable blindness accounting to $63.7 \% .{ }^{1}$ Delayed reporting for treatment leading to ophthalmic emergency like lensinduced glaucoma (LIG), a type of secondary glaucoma remains one of the most important cause of irreversible visual loss, especially so in the rural population.

Lens induced glaucoma may occur as open angle or secondary closed angle glaucoma. Intumescent cataractous lens, can block the pupil resulting in acute secondary angle closure glaucoma (phacomorphic glaucoma). Secondary open angle glaucoma can be 'Phacolytic glaucoma' occurring in patients with hypermature cataract where the trabecular meshwork is blocked by leakage of lens protein through an intact capsule along with macrophages, 'lens particle glaucoma' due to liberated fragments of lens material with a grossly disrupted lens capsule which can occur cataract surgery, trauma or Nd YAG laser capsulotomy or 'Phacoantigenic glaucoma' due to immune response to lens protein antigen. ${ }^{2-6}$

It is a clinical condition characterized by severe secondary glaucoma (mimicking acute angle closure glaucoma) in one eye with intumescent cataract, senile mature cataract or hyper mature senile cataract yet with an open angle with normal intraocular pressure and open angle in other eye, and a prompt relief of symptoms and restoration of vision after cataract extraction in the effected eye.

LIG is also associated with ectopia lentis. ${ }^{6}$ The dislocated lens in such situations can elevate the IOP through various mechanisms. Traumatic LIGs are associated with lens subluxation/dislocation, lens rupture or rarely a hypersecretory glaucoma. ${ }^{7}$

Despite increase in quality eye care services, the cataract backlog is increasing due to various barriers in the uptake of cataract surgery resulting in increased incidence of lens induced glaucoma. Hence this study was undertaken to evaluate the clinical profile and visual outcome following management of lens induced glaucoma.

\section{Materials and Methods}

This retrospective non-randomized, hospital based study was approved by Institutional Ethics Committee and abided by the tenets of declaration of Helsinki. The study included all patients diagnosed as lens induced glaucoma, who underwent cataract surgery over a five year period. We excluded cases of congenital cataract, secondary cataract, complicated cataract, and known cases of glaucoma from this study. Medical records of these patients were reviewed for collection and analysis of data.

Procedure: All patients were subjected to complete ophthalmic examination with preoperative corrected visual acuity (CDVA), intraocular pressure (IOP) measurement by non-contact tonometry, detailed anterior and posterior segment evaluation, Keratomerty and A scan biometry with SRK 2 formula.

Informed consent were taken from all patients who underwent emergency preoperative reduction in intraocular pressure by administering intravenous 
infusion of 100ML mannitol 20\%, oral acetazolamide $250 \mathrm{mg}$ and topical beta blocker timolol $0.5 \%$ eye drops twice daily followed by cataract surgery and combined surgery under peribulbar anaesthesia. Anterior vitrectomy was done in needed patients and any intraoperative complications were noted.

Postoperative medications include an antibiotic steroid eye drops used for 6 weeks in a tapering dose. All patients were followed from $1^{\text {st }}$ day, $1^{\text {st }}$ week, $1^{\text {st }}$ month and $3^{\text {rd }}$ month and at each visit patient were evaluated for CDVA, IOP and any postoperative complications.

Statistical Methods Used: Data was entered into Microsoft excel data sheet and analyzed using SPSS 22 version software (IBM SPSS Statistics, Somers NY, USA). Categorical data was represented by frequencies and proportions. Continuous data is represented as mean and standard deviation. Paired $\mathrm{t}$ test is the test of significance for paired quantitative data and $p$ value (Probability that the result is true) of $<0.05$ was considered as statistically significant after assuming all the rules of statistical tests.

\section{Results}

This study included 48 eyes out of which $23(47.9 \%)$ were males and 25(52.1\%) females aged between 50 to 80 years. The mean age of the patients was $55.56 \pm 14.8$ years and the mean duration of symptoms was $3.38 \pm$ 1.606 days.

Table 1 shows the type of lens induced glaucoma. In our study 32(66.7\%) cases had presented with phacomorphic glaucoma, 13 (27.1\%) Phacolytic glaucoma, Traumatic dislocated lens $2(4.2 \%)$ and $1(2 \%)$ case with lens particle glaucoma.

Fig. 1 shows the duration of symptoms like pain, redness, headache, diminution of vision, photophobia and reporting to the hospital. 5 cases reported immediately while $9,16,7$ and 4 patients on $2^{\text {nd }}, 3^{\text {rd }}, 4^{\text {th }}$ and 1 week respectively.

Fig. 2 shows the preoperative and postoperative IOP. In the study Mean pre-operative IOP was $35.60 \pm$ $5.81 \mathrm{mmHg}$ and postoperative IOP was $20.417 \pm 2.33$. There was significant decrease in IOP after successful cataract and combined surgery when compared to preoperative values $(\mathrm{P}<0.001)$.

Table 2 shows the treatment options where 40 patients had undergone small incision cataract surgery and phacoemulsificaton, 6 patients combined surgery due to persistent high IOP even after anti glaucoma medications and 2 cortical wash.

Fig. 3 shows the preoperative and postoperative vision of patients. In this study the mean preoperative Visual acuity was $1.44 \pm 0.76$ and postoperative visual acuity was $0.34 \pm 0.20$.

There was significant improvement in postoperative visual acuity ( $\mathrm{P}<0.001)$. Postoperatively $31(64 \%)$ of the patients gained good vision, better in $12(25 \%)$ and Low vision in $5(10.4 \%)$ cases.

Fig. 4 reveals the intraoperative and postoperative complications observed in this study. The most common intraoperative complication noted was shallow anterior chamber in $12(25 \%)$ eyes followed by posterior capsular rupture (PCR) in $5(10.4 \%)$ and vitreous loss in 3(6.3) eyes. Striae keratopathy was the early postoperative complications noticed in $14(29.2 \%)$ eyes, Iritis in 10 (20.8\%) eyes and the late complications were Cystoid macular edema in $2(4.16 \%)$ patients.

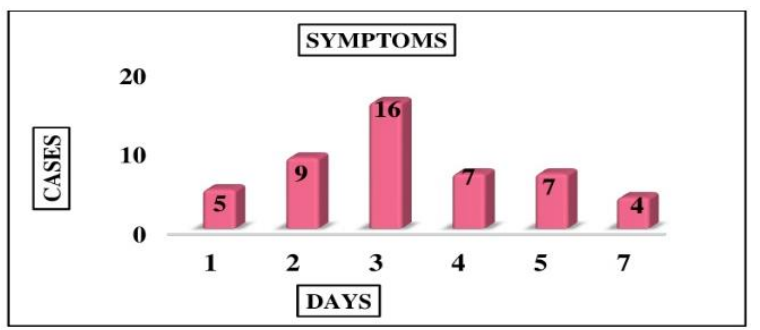

Fig. 1: Bar diagram showing duration of symptoms among the patients

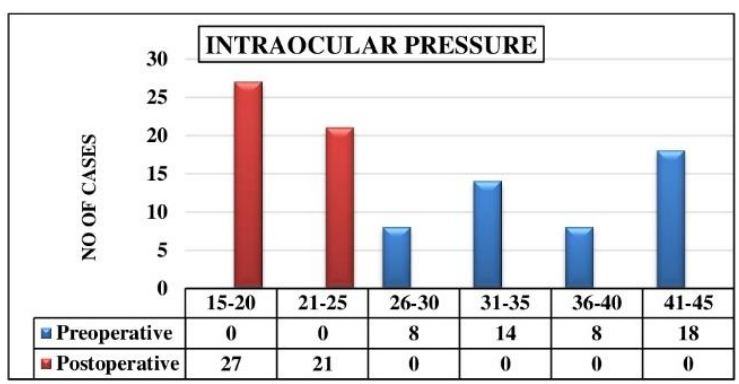

Fig. 2: Bar diagram showing preoperative and postoperative intraocular pressure

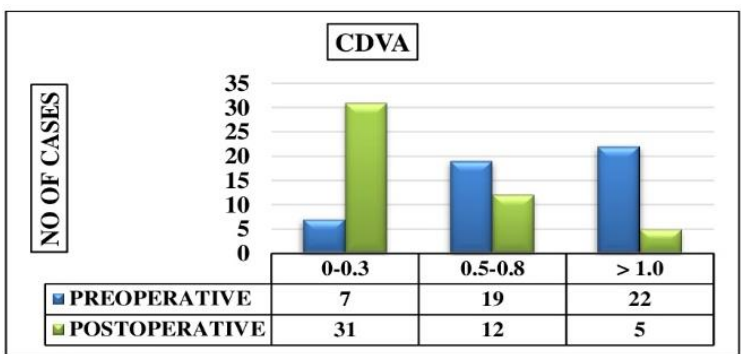

Fig. 3: Bar diagram showing preoperative and postoperative CDVA



Fig. 4: Bar diagram showing intraoperative and postoperative complications 
Table 1: Type of lens induced glaucoma

\begin{tabular}{|l|c|c|c|}
\hline \multicolumn{1}{|c|}{ Type } & Male & Female & Total \\
\hline Phacomorphic glaucoma & 14 & 18 & $32(66.7 \%)$ \\
\hline Phacolytic glaucoma & 6 & 7 & $13(27.1 \%)$ \\
\hline Traumatic dislocated lens & 2 & 0 & $2(4.2)$ \\
\hline Lens particle glaucoma & 1 & 0 & $1(2)$ \\
\hline Total & 23 & 25 & 48 \\
\hline
\end{tabular}

Table 2: Various treatment options

\begin{tabular}{|l|c|c|c|c|c|c|}
\hline \multirow{2}{*}{ Treatment options } & \multicolumn{2}{|c|}{ Male } & \multicolumn{2}{c|}{ Female } & \multicolumn{2}{c|}{ Total } \\
\cline { 2 - 8 } & $\mathbf{N}$ & $\mathbf{\%}$ & $\mathbf{N}$ & $\mathbf{\%}$ & $\mathbf{N}$ & $\mathbf{\%}$ \\
\hline Cataract extraction with PC IOL implantation & 19 & 90.5 & 21 & 77.8 & 40 & 83.3 \\
\hline $\begin{array}{l}\text { Trabeculectomy + Cataract extraction with PC } \\
\text { IOL implantation }\end{array}$ & 2 & 9.5 & 4 & 14.8 & 6 & 12.5 \\
\hline Cortical wash & 0 & - & 2 & 7.4 & 2 & 4.2 \\
\hline Total & 21 & 100 & 27 & 100 & 48 & 100 \\
\hline
\end{tabular}

\section{Discussion}

Most cases of lens induced glaucoma occurs in patients with a longstanding mature and hypermature cataract associated with history of severely reduced vision in one eye leading to severe ocular complications like increased inflammation and intraocular pressure.

In this study, the mean age of the patients was 55.56 \pm 14.8 years and females seemed to have an increased risk of LIG compared to male, $50.1 \%$ and $47.9 \%$ respectively. Other studies also showed similar results. ${ }^{3,8,9}$ the reason being dependency on other family members financially and physically.

This entity was more common in females because of socio-economic constraints.

Most frequent type of LIG observed was Phacomorphic glaucoma (66.7\%) followed by Phacolytic glaucoma $(27.1 \%)$ which was comparable with studies. ${ }^{3,8}$ This clearly reveals the negligence and lack of awareness about the significance of early management in the prevention of blindness. [Table 1]

Majority of the patients reported after 3 days seeking symptomatic relief and unaware of the severity of cataract and its complication. [Fig. 1] Pradhan et al in his study found $70 \%$ of cases presented after 10 days of onset of symptoms. The reasons were financial constraints and lack of support. We attributed to socio economic constraints prevalent in rural population.

The mean preoperative and postoperative CDVA was $1.44 \pm 0.76$ and $0.34 \pm 0.20$ respectively. The improvement in postoperative visual acuity was statistically significant. Markedly reduction in visual acuity in all cases was due to cataract as well as due to corneal edema secondary to a sudden rise of intraocular pressure. 31(64\%) patients gained good vision, better in $12(25 \%)$ and low vision in $5(10.4 \%)$ which was due to cystoid macular edema, age related macular degeneration and glaucomatous optic atrophy. [Fig. 3]. Results were similar to study conducted by Murthy et $\mathrm{al}^{10}$ and better than Pant Sitoula et al. ${ }^{11}$

The mean pre-operative intraocular pressure was $35.60 \pm 5.81 \mathrm{mmHg}$ and postoperative IOP was 20.417 \pm 2.33 . [Fig. 2]. Eighteen cases presented with IOP more than $40 \mathrm{mmHg}$ and 22 above $30 \mathrm{mmHg}$. After successful preoperative hypotensive therapy and surgical management IOP decreased significantly providing symptomatic relief to patients similar to the study done by Jyoti Pant Sitoula et $\mathrm{al}^{11}$ and Bhuyan et al. ${ }^{12}$ IOP control was achieved with acetazolamide $500 \mathrm{mg}$ oral three times a day, topical timolol maleate $0.5 \%$ twice daily, intravenous mannitol $20 \%$ with a dose of $5 \mathrm{ml} / \mathrm{kg}$ was given over 30 minutes in cases where intraocular pressure (IOP) was above $40 \mathrm{mmHg}$ prior to the cataract surgery. Topical steroids were instilled to reduce inflammation in traumatic lens induced and phacolytic glaucoma.

The most common intraoperative complication noted was mild corneal edema in $12(25 \%)$, shallow anterior chamber in $12(25 \%)$ eyes followed by posterior capsular rupture (PCR) in $5(10.4 \%)$ and vitreous loss in 3(6.3) eyes. Striae keratopathy was the early postoperative complications noticed in 14 (29.2\%) eyes, Iritis in $1020.8 \%$ ) eyes that subsided with prompt postoperative medications and the late complications were Cystoid macular edema in $2(4.16 \%)$ patients [Fig. 4] comparable to study by R. Kothari. ${ }^{9}$ But postoperative uveitis was the most frequently encountered complication observed by Pant Sitoula et al. ${ }^{11}$ in about $26 \%$ of postoperative patients and elevated IOP resulting in optic atrophy was seen in 5 patients $(12.5 \%)$.

The definitive treatment for lens induced glaucoma is cataract extraction and the outcome depends on the duration between the onset of symptoms and the treatment. Cataract surgery was performed in all patients but combined trabeculectomy was performed on 6 patients due to persistent high IOP and this was successful in reducing the IOP postoperatively during 3 
months' follow-up visit. [Table 2] Angra et al ${ }^{13}$ have recommended combined surgery in patients with longer duration of attack ( $>7$ days) but a recent study by Senthil et al. ${ }^{14}$ has shown that $97 \%$ of their cases had IOP $<21$ $\mathrm{mmHg}$ at 6 months follow up following cataract surgery alone and had a faster visual recovery than combined group and hence recommends cataract surgery alone.

\section{Conclusion}

LIG is an important vision-threatening disease occurring in the elderly due delayed treatment of mature cataract. Lack of awareness of this condition is the main reason for people becoming blind inspite of easy availability of surgical facilities. This study shows that it is important to conduct periodic screening of the elderly to identify cataracts and manage it accordingly to prevent irreversible blindness.

\section{Conflict of Interest: None}

\section{References}

1. Government of India, National Survey on Blindness and Visual Outcome after Cataract Surgery, 2001-2002, vol. 77, National Programme for Control of Blindness, Ministry of Health, Government of India, New Delhi, India, 2002.

2. Ellant JP, Obstbaum SA. Lens-induced glaucoma. Doc Ophthalmol. 1992;81(3):317-38.

3. Pradhan D, Henning A, Kumar J, Foster A. A prospective study of 413 cases of lens-induced glaucoma in Nepal. Indian J Ophthalmol. 2001;49:103-7.

4. Kanski J, Brad Bowling. Lens-related glaucoma. In: kanski J, Brad Bowling, eds. Clinical Ophthalmology: A systematic approach. 7th ed. Elsevier Saunders; 2011.

5. Sowka J. Phacomorphic glaucoma: case and review. Optometry. 2006;77(12):586-9.

6. Papaconstantinou D, Georgalas I, Kourtis N, Krassas A, Diagourtas A, Koutsandrea C, Georgopoulos G. Lensinduced glaucoma in the elderly. Clin Interv Aging. 2009;4:331-6.
7. Bai HQ, Yao L, Wang DB, Jin R, Wang YX. Causes and treatments of traumatic secondary glaucoma. Eur J Ophthalmol 2009;19:201-6.

8. Rijal AP, Karki DB (2006). Visual outcome and IOP control after cataract surgery in lens induced glaucomas. Kath Univ Med J. 2006;4:30-3.

9. Raghunandan Kothari, Sandeep Tathe, Pratik Gogri, and Akshay Bhandari. Lens-Induced Glaucoma: The Need to Spread Awareness about Early Management of Cataract among Rural Population. ISRN Ophthalmology. Vol.2013: ArticleID581727,1-3, 2013.

10. Srinivasa Murty, V. Murali Krishna, Beatrice Choppara. A Clinical Study of Lens Induced Glaucoma. International Journal of Scientific Research and Management. 2015;3:2535-38.

11. Rakshya Pant Sitoula, Indrajit Sarkar, Devdutta Nayak, Sanjay Kumar Singh. Lens induced glaucoma: An experience in tertiary eye care center in eastern Nepal. Nepal J Ophthalmol. 2016;8(16):161-166.

12. Bhuyan J, Bharali D M. Management of "Lens Induced Glaucoma"-Aclinical Study. Journal of Dental and Medical Sciences. 2016;15:76-82.

13. Angra SK, Pradhan R, Garg SP (1991). Cataract induced glaucoma - an insight into management. Indian $J$ Ophthalmol. 1991;39:97-101.

14. Senthil S, Chinta S, Rao HL, Choudhari NS, Pathak-Ray $\mathrm{V}$, Mandal AK, et al. Comparison of cataract surgery alone versus cataract surgery combined with trabeculectomy in the management of phacomorphic glaucoma. J Glaucoma. 2016;25:209-13.

How to cite this article: Jayakumar S, Krishnappa K, Darshan SM, Meghana C.R.G, Laxman B. H. Clinical profile, visual outcome and complications after cataract surgery in patients with lens induced glaucoma. Indian $\mathrm{J}$ Clin Exp Ophthalmol. 2018;4(4):511-514. 